\title{
Miranda
}

Revue pluridisciplinaire du monde anglophone /

Multidisciplinary peer-reviewed journal on the English-

speaking world

19 | 2019

Rethinking Laughter in Contemporary Anglophone Theatre

\section{The Scarlet Letter : A comme adaptation}

Critique

\section{Aliette Ventéjoux}

\section{(2) OpenEdition}

\section{Journals}

Édition électronique

URL : http://journals.openedition.org/miranda/21177

DOI : 10.4000/miranda.21177

ISSN : 2108-6559

Éditeur

Université Toulouse - Jean Jaurès

\section{Édition imprimée}

Date de publication : 7 octobre 2019

\section{Référence électronique}

Aliette Ventéjoux, «The Scarlet Letter: A comme adaptation », Miranda [En ligne], 19 | 2019, mis en ligne le 11 octobre 2019, consulté le 16 février 2021. URL : http://journals.openedition.org/miranda/21177 ; DOI : https://doi.org/10.4000/miranda.21177

Ce document a été généré automatiquement le 16 février 2021.

\section{(c)}

Miranda is licensed under a Creative Commons Attribution-NonCommercial-NoDerivatives 4.0 International License. 


\section{The Scarlet Letter : A comme adaptation}

Critique

Aliette Ventéjoux

\section{Informations sur la pièce}

Lieu: Théâtre de la Colline. Représentation du 15 janvier 2019

Mise en scène, texte, scénographie, costumes et jeu : Angélica Liddell

Librement inspiré du roman de Nathaniel Hawthorne

Comédiens, comédiennes : Joele Ansastasi, Tiago Costa, Julian Isena, Angélica Liddell, Borja López, Tiago Mansilha, Daniel Matos, Eduardo Molina, Nuno Nolasco, Antonio Pauletta, Antonio L. Pedraza, Sindo Puche

Et la participation de Mathias Caroff, Thomas Sgarra, Philomène Troullier

Assistanat à la mise en scène : Borja Lopez

Lumières : Jean Huleu

Son : Antonio Navarro

Traduction : Christilla Vasserot

Production et diffusion : Gumersindo Puche

Communication : Génica Montalbano

Régie : Frédéric Gourdin

Régie vidéo : Igor Minosa

Régie lumières : Stéphane Touche

Technicien lumières : Pascal Levesque

Régie son : Émile Denize

Technicien HF : Kévin Cazuguel

Machinistes : Farid Aberbour, Nicolas Gérard et Antoine Mary

Habilleuse : Sonia Constantin

Accessoiriste : Julie Berce 
Durée $1 \mathrm{~h} 40$

Spectacle en anglais, espagnol, italien, portugais, surtitré en français.

\section{Liens}

La Colline Théâtre National : https://www.colline.fr/

\section{Critique}

En 1850, Nathaniel Hawthorne publie The Scarlet Letter, qui retrace l'histoire d'Hester Prynne dans le Boston puritain du XVII ${ }^{\mathrm{e}}$ siècle. Hester, coupable d'adultère, est marquée du sceau de la lettre A qui rappelle son crime à ses concitoyen-nes. Chez Angélica Liddell, dont la pièce du même nom est très librement adaptée du roman de Hawthorne, la lettre A est davantage le A de l'art, de l'artiste, et, bien évidemment, le A d'Angélica.

4 Le texte de Hawthorne devient prétexte à une représentation, une performance durant laquelle se succèdent de nombreux tableaux. Sur scène, les comédien-nes hurlent, courent, se dénudent, chantent, et les langues se mélangent. Le texte est déclamé en anglais, espagnol, italien, portugais, et surtitré en français, ce qui renforce l'impression d'être submergé par ce qui se passe sur scène. S'y ajoute de la musique, baroque - Lully par exemple - mais également des chants liturgiques, ou encore «I put a spell on you » de Screamin' Jay Hawkins, et même une chanson bien connue du groupe O-Zone. Sans oublier les cris, ceux des hommes qui se trouvent sur scène, ou d'Angélica, qui relatent la violence et la souffrance dont il est question. Les couleurs et la lumière renforcent également cette impression, car la scène est la plupart du temps d'une couleur rouge plus ou moins écarlate, parfois aussi jaune, ou encore noire, à l'image des costumes que portent les comédiens au début de la pièce.

5 Celle-ci s'ouvre sur Adam et Ève, nus, qui se recueillent sur une tombe portant le nom de Hawthorne. Le péché originel est d'emblée suggéré, et ce « prologue » nous rappelle par ailleurs le début du roman de Hawthorne: "The founders of a new colony, whatever Utopia of human virtue and happiness they might originally project, have invariably recognized it among their earliest practical necessities to allot a portion of the virgin soil as a cemetery, and another portion as the site of a prison. " ${ }^{1} \mathrm{Si}$ Liddell a fait le choix de faire figurer la tombe et donc de rappeler le cimetière, la présence des deux corps nus fait écho au péché d'Hester dans le roman, ainsi qu'à la prison et à sa punition pour avoir fauté. 
Fig. 1

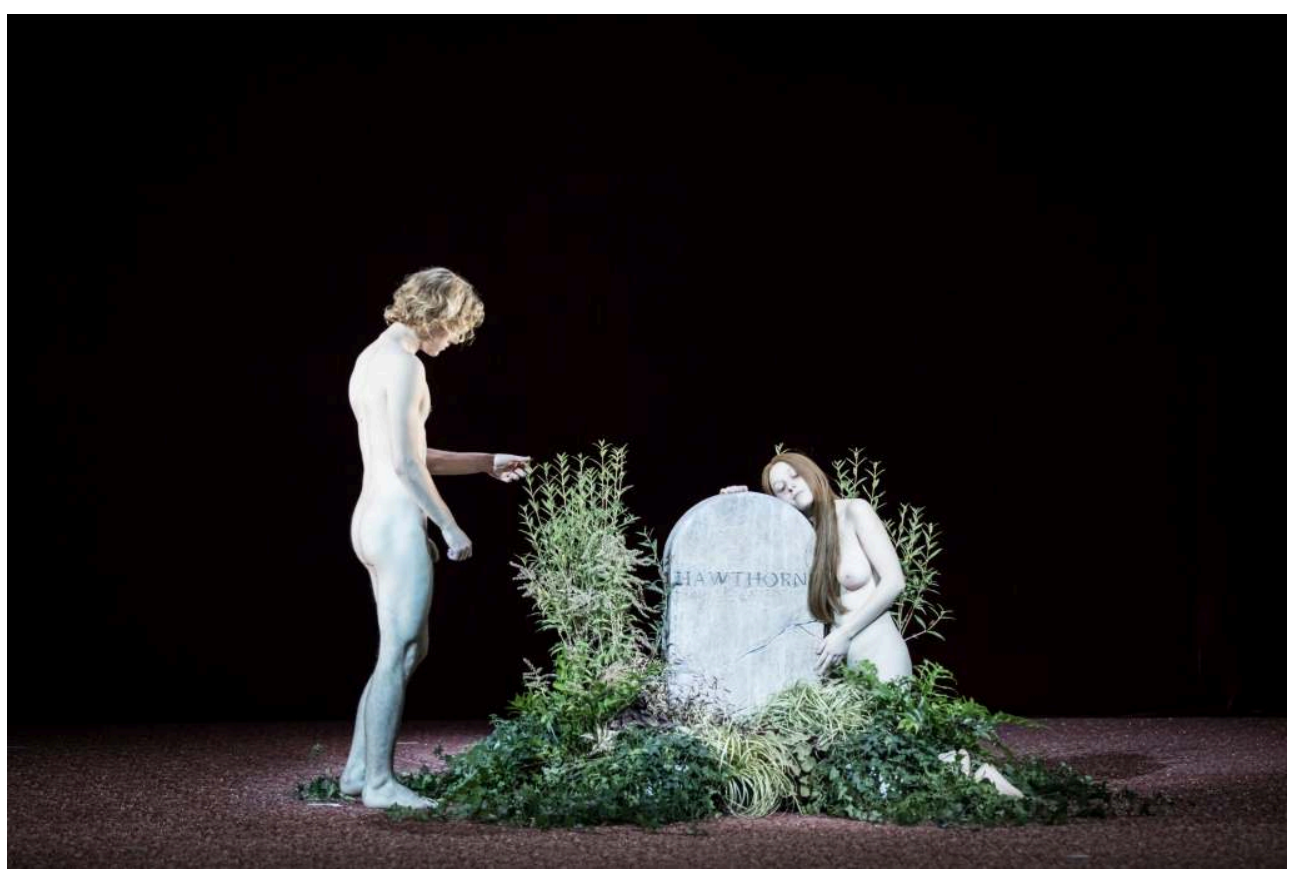

Crédit : Simon Gosselin

6 Les deux acteurs sont ensuite remplacés par Angelica/Hester, qui porte une large robe noire et dont le dos est strié de cicatrices, témoins de sa faute. Puis entrent sur scènes des hommes vêtus de noirs et portants des cagoules pointues qui ne sont pas sans rappeler celles du Ku Klux Klan par exemple. Mais ils ne gardent pas longtemps leur costume, et se retrouvent vite nus. Ils le resteront pendant toute la pièce. Si l'on peut s'interroger sur les raisons de ce choix, on finit rapidement par oublier qu'ils sont nus. Enfin presque, car la mise en scène nous le rappelle de temps à autre, notamment lorsque l'auteure joue sur scène avec leurs sexes. Malgré cette nudité constante, le spectacle ne peut être qualifié d'obscène. Plus que dérangeante, la nudité de ces hommes peut même finir par être lassante.

7 Si les questions de la place de la femme et de l'emprise du patriarcat se posent, les réponses apportées restent provocatrices, à l'image de ces corps nus, et, dans une certaine mesure, un peu contradictoires. En effet, l'un des monologues d'Angélica Liddell semble être une virulente critique du mouvement MeToo, durant laquelle elle s'attaque aux femmes de plus de 40 ans et au dégoût provoqué par leur corps. Ses propos sur "l'odeur de pisse » et les "vagins fanés " ${ }^{2}$ ne sont pas moins violents, et la question du second degré demeure, difficile à trancher. À ce moment là de la pièce, le malaise est certain et il devient difficile d'adhérer aux propos: «Je n'aime pas ce monde où les femmes ont cessé d'aimer les hommes. Je n'aime pas ce monde où les femmes haïssent les hommes. Je ne l'aime pas. Je n'aime pas être une femme parmi les femmes. Je n'aime pas ça. $»^{3}$

8 Tout comme Hester Prynne, Angelica Liddell détourne la lettre. Grâce à son savoirfaire, Hester brode et enjolive le A : « À distraire ainsi la lettre, la femme condamnée se soustrait à la loi, à la loi appliquée à la lettre, et l'exhibe comme une forme inavouée de spectacle. Elle met en évidence l'antinomie constitutive du régime théocratique : tandis que la représentation théâtrale est par principe bannie du Nouveau Monde, le pouvoir 
ne peut pas ne pas manifester sa puissance par une forme détournée de mise en scène. Le spectacle du châtiment, les processions religieuses et les défilés militaires sont les seules formes de fêtes tolérées par les puritains qui interdisent les survivances des festivités populaires. $»^{4}$ La mise en scène de Liddell, qui a elle aussi tour à tour des airs de spectacle du châtiment ou de processions religieuses, souligne le détournement du médium "théâtre ». Pas d'unité, que de la nudité. Ou plutôt si. Une unité subsiste, construite autour de l'art.

Et c'est aussi celle-ci qui tisse un lien entre la pièce et le texte, à la manière dont Hester tisse et brode : "On the breast of her gown, in fine red cloth, surrounded with an elaborate embroidery and fantastic flourishes of gold thread, appeared the letter A. It was so artistically done, and with so much fertility and gorgeous luxuriance of fancy, that it had all the effect of a last and fitting decoration to the apparel which she wore; and which was of a splendor in accordance with the taste of the age, but greatly beyond what was allowed by the sumptuary regulations of the colony. $»^{5}$ L'art seul permet à Hester et Angélica de s'exprimer, et de se faire entendre. Si Hester tire sur les fils de sa broderie, Angélica Liddell, quant à elle, telle une prêtresse, tire sur les fils qui retiennent les hommes prisonniers, littéralement.

Fig. 2

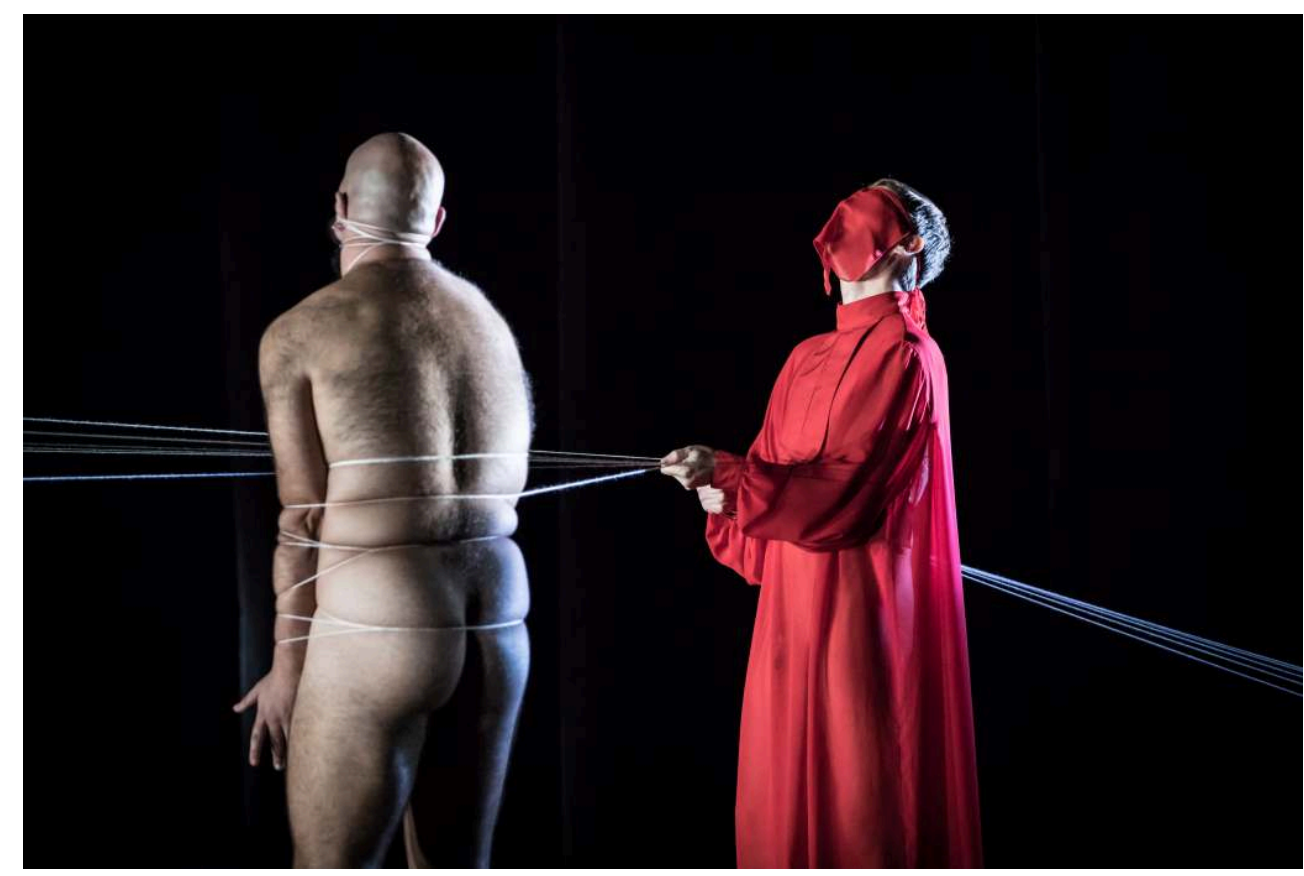

Crédit : Simon Gosselin

Le monologue final, plus puissant nous semble-t-il que le précédent, recentre l'attention sur le A de l'artiste tout en défendant la liberté absolue de la création. L'énumération des noms des artistes, auteurs et penseurs est un moment bouleversant. Les propos de Liddell questionnent le monde d'aujourd'hui, qu'il s'agisse du féminisme ou de la place de l'art et de l'artiste. Sans juge en effet, pas d'art: « Celle qui vous parle tue, vole, pervertit. Sans juge, l'art n'existerait pas. Sans moralisme, l'art n'existerait pas. Sans hypocrisie, l'art n'existerait pas. Bref, sans vous, l'art n'existerait pas. Je vous remercie donc de me mépriser. » Ces mots, énoncés au début de la pièce, annoncent les transgressions et la provocation à venir. Liddell se pose en quelque sorte en sorcière, 
mais une sorcière bien différente de celles dont il est question dans le dernier ouvrage de Mona Chollet ${ }^{6}$.

11 Le A est donc finalement aussi le A de Artaud, le A de l'artiste auquel on revient dans ce dernier monologue, qui, après les provocations de certains propos et de certaines scènes, rappelle aux spectateurs que l'important reste la liberté absolue de la création. Si cette pièce questionne le monde d'aujourd'hui, pas si différent finalement du monde puritain décrit par Hawthorne, elle est avant tout un plaidoyer en faveur de l'art et de la transgression qu'il permet, de l'absolue nécessité qu'une société a de l'art et de ses artistes.

\section{NOTES}

1. Nathaniel Hawthorne. The Scarlet Letter and Other Writings. New York, London: W. W. Norton \& Company. 2005. 36.

2. Angélica Liddel. The Scarlet Letter.

3. Ibid.

4. Michel Imbert. « S.L. : l'esprit de la lettre et les spectres de l'Amérique fantôme. » In Nathaniel Hawthorne, The Scarlet Letter. Bruno Monfort (Ed.). Paris : Éditions du Temps. 2005. pp.116-159. 120.

5. Nathaniel Hawthorne. Op. Cit. 40.

6. Mona Chollet. Sorcières. La puissance invaincue des femmes. Paris : Éditions la Découverte. 2018.

\section{RÉSUMÉS}

Compte rendu de la pièce d'Angélica Liddell The Scarlet Letter librement inspirée du roman de Nathaniel Hawthorne.

Performance review Angélica Liddell's play The Scarlet Letter, adapted from Nathaniel Hawthorne's novel.

\section{INDEX}

Mots-clés : adaptation, Angélica Liddell, art, féminisme, Nathaniel Hawthorne, lettre, monologue, performance, provocation, puritanisme, théâtre, transgression

Keywords : adaptation, Angélica Liddell, art, feminism, Nathaniel Hawthorne, letter, monologue, performance, provocation, puritanism, theatre, transgression

Thèmes: Theater 
AUTEUR

\section{ALIETTE VENTÉJOUX}

Docteur

Université Paris II - Panthéon Assas

alietteventejoux@protonmail.com 